\title{
DETERMINING FACTORS OF FISH PRODUCTION IN EGYPT
}

Amr Feteha Hanafy Mahmoud, Faculty of Commerce, Alexandria University, Egypt, Human and Social Sciences compound - Suter St. El Shatby- Alexandria, Egypt

dx.doi.org/10.18374/JIBE-20-3.1

\begin{abstract}
The fish production in Egypt is failing to cover the increasing in consumption needs, so the research aims to determine the factors that influence fish production in Egypt during 1990-2016. We were able to identify key determinants of fish production; experimented by Simultaneous equations model (SEM). The estimate suggests that fish production is positively associated with fish consumption; fish production requirement costs; fishery cooperative associations; and fish stock. As well as, negatively associated with fish price and fishers. The current study enabled us to evaluate factors affecting fish production. Policymakers should have the interest to observe the outcomes and recommendations of this study for optimal policy planning.
\end{abstract}

Keywords: Fish production - Capture fisheries - Cultured fisheries - Fish consumption - Fish gap Simultaneous equation- Fiscal policy. 